Reiner Hil debrandt

\title{
KULTURGESCHICHTLICHE ASPEKTE DES WORTSCHATZES IM SUMMARIUM HEINRICI
}

Ich habe über die kulturgeschichtlichen Aspekte des Wortschatzes im Summarium Heinrici (1974/1982/1995) zu berichten und muss dazu einleitend drei grundsätzliche Problemkomplexe thematisieren:

Als Erstes stellt sich die Frage: Ist es ein W ortschatz des 11. oder des 12. J ahrhunderts? Der mit mir in fruchtbarer Auseinandersetzung befindliche Kollege W egst ein (1985) hat trotz des spätalthochdeutschen Lautstandes der deutschen Glossenwörter vehement für die Entstehung des Summariums erst im 12. J ahrhundert plädiert und dafür als Beweis die Seneca-Belesenheit des Summarium-Verfassers ins Feld geführt. Dieser beginnt nämlich seinen Prosaprolog mit einer wortwörtlichen Argumentation aus Senecas 33. Brief an Lucilius und übernimmt aus dem 39. Brief auch noch die ureigene Wortprägung Senecas „Summarium" als Titel für sein eigenes Werk. Da nun nach einer einschlägigen Untersuchung des Engländers L. D. Reynolds (1965) die noch für die Karolingerzeit gut nachweisbare Seneca-Belesenheit nach zwei dunklen J ahrhunderten in Deutschland erst wieder im 12. J ahrhundert einsetzen soll, müsse - so W egst ein - auch der Summarium-Verfasser erst im 12. J ahrhundert angesiedelt werden. ${ }^{1}$ Misslich an dieser Argumentation war nur, dass der Verfasser den Namen selbst - Seneca - gar nicht genannt hatte, sondern die Floskel gebrauchte: „ut quidam sapiens ait“ - ein gewisser Weiser also -, eine Autorität zwar, aber noch keine, die vom Namen her unverwechselbar gewesen wäre. Das spricht nun aber - wie ich meine ( $\mathrm{H}$ il debr andt

\footnotetext{
1 Reynol ds hat in einem jüngeren Beitrag (in: Texts and Transmission, a Survey of the Latin Classics. Oxford 1983, S. 369-375) unter Berufung auf B. B isch of $f$ die Position Westdeutschlands hinsichtlich der Verbreitung von Seneca-Handschriften aufgewertet. Außerdem hat Reynolds diesbezüglich auch das Buch von Klaus-Dieter Nothdurft: Studien zum Einfluss Senecas auf die Philosophie und Theologie des zwölften J ahrhunderts. Leiden/K öln 1963, nicht zur K enntnis genommen.
} 
1986) - entschieden dafür, dass der Verfasser doch noch dem 11. J ahrhundert angehörte, und besonders dann, wenn er auch noch in dem traditionsreichen K loster Lorsch lokalisiert werden kann, wofür es gute, aber hier nicht zu erörternde Argumente gibt (H il debr andt 1995, Spalte 517).

Zweitens das Problem der Zweisprachigkeit als unausgesprochener Intention des Verfassers: Der Verfasser sagt in dem erwähnten Prolog, obwohl er dort die originelle inhaltliche Konzeption der elf Bücher seines Summariums als Lehrbuch für den Elementarunterricht kurz aber prägnant beschreibt, mit keinem Wort etwas über die mehr als 3000 deutschen Wörter, die er als integrale Bestandteile in sein Werk einbringt, sodass der Charakter einer gewollten Zweisprachigkeit dieses Lehrbuches doch wie ich meine - erstmals voll zum Tragen kommt. Wenn er die deutschen Wörter deshalb nicht für erwähnenswert hielt, weil er sie nach vorausgehendem U sus immer noch als echte Glossen, $d$. h. Verständnishilfen für das $L$ atein verstand, so haben sich die Rezipienten seines Werkes im deutlichen Gegensatz dazu doch sehr entschieden von dieser Übergewichtung des $L$ ateins distanziert und das W erk gerade wegen seiner Aufwertung der Volkssprache Deutsch zu schätzen gewusst. Das beweist ganz offensichtlich die Auslassung des rein lateinischen ersten Viertels über die Grammatik in den meisten Handschriften der ersten Fassung und das völlige Fehlen dieses Teilstückes in der zweiten Fassung, in der auch die restlichen drei Viertel noch einmal um die Hälfte zusammengestrichen wurden, und zwar ganz gezielt auf Kosten des Lateins und zu Gunsten der deutschen Wörter. Über die Datierung dieser zweiten Fassung (Redaktion B) gibt es bei W. W egst ein (1985, S. 84ff.) aufgrund eines angeblichen terminologischen Indizes die irrige Fixierung auf die Mitte des 14. J ahrhunderts. Ich habe dem alsbald widersprochen (H il debr andt 1986) und finde meine Datierung im frühen 12. J ahrhundert inzwischen durch Hildegard von Bingen bestätigt, deren sog. „Lingua ignota“ in engster Abhängigkeit zu dieser zweiten Fassung des Summariums entstanden ist ( $\mathrm{H}$ il debrandt 1996a), deren Entstehungsort übrigens aufgrund einer gemeinsamen Wortgleichung mit der Trierer Handschrift Trier sein könnte. ${ }^{2}$

Es handelt sich um die Glossierung von veternosus antiquus decrepitus mit altziergerner, die nur in der Trierer Handschrift $C$ der ersten Fassung des Summariums und in der Leithandschrift $D$ der zweiten Fassung vorhanden ist und von mir im Registerband des Summariums (Band 3, S. 5) noch falsch gedeutet wurde. Erst durch das bei Hildegard von Bingen belegte Adjektiv ziergerne 'prunksüchtig, eitel' ist auch die eigenwillige Wortbildung des Summariums hinreichend durchsichtig als 'eitler alter (Mann)' (H il debr andt 1996b, S. 130-133). 
Drittens der traditionsgeschichtliche Zielgruppenwechsel: Die im originären Prosaprolog angesprochene Zielgruppe dürfte eindeutig im K losterschulenmilieu anzusiedeln gewesen sein. Nun gibt es aber einen nur von zwei süddeutschen Handschriften $(A B)$ tradierten zweiten Prolog in Versen, der wegen seiner Künstlichkeit, d. h. Hexametern mit Akro-/Meso-/ Telestichon - zu lesen als „H einrici Summarium id est" - , eindeutig ins 12. J ahrhundert gehört und dem Werk durch diese Aufbesserung - auch hinsichtlich des erst hier eingebrachten und deshalb nicht originären Verfassernamens - den erweiterten Verwendungsbereich in den KathedralschuIen verschafft haben dürfte. In den beiden Handschriften findet sich daher auch mindestens ein interpolationsverdächtiges Kapitel im Rahmen des rein lateinischen Grammatikteils (II, 12 „De cognoscendis primis sillabis per exemplum" ), das eine lange, alphabetisch geordnete Wörtersammlung in Hexametern bietet, was so gar nicht zum Charakter der sonstigen K onzeption des Summariums passt. Den letzten Sprung von den Kathedralschulen in die Universitäten hat das Summarium allerdings nicht geschafft. Dort erzielte das Prestige der adaptierten Gelehrsamkeit von Italien und $\mathrm{Frankreich}$ her einen höheren Stellenwert gegenüber dem bodenständigen Eigenwuchs des Summariums. In den spätmittelalterlichen $V_{0}$ kabularien kam dieses so gewonnene höhere kulturelle Niveau voll zum Tragen und verwehrte dem Summarium damit eine kontinuierliche Fortwirkung.

Nach diesen Präliminarien nun die gezielte Blickrichtung auf das Thema: Generell kulturgeschichtlich positioniert ist das Summarium eine mit eigener Konzeption umgestaltete Bearbeitung der "Etymologien“ des Isidor von Sevilla (1911). Dieses W erk hatte bekanntlich die "septem artes liberales" nur einleitend und ungleichgewichtig im Blick: Schon im Trivium waren Rhetorik und Dialektik gegenüber der Grammatik umfangsmäßig schlechter weggekommen. Im Summarium erscheinen diese beiden nur resthaft als Anhängsel zur Grammatik und vom bei Isidor noch gut vertretenen Quadruvium fehlen wiederum die beiden mathematischen Disziplinen. Sodann gibt es von der Musik und der Astronomie nur einige praxisorientierte Teilbereiche, wobei sich bei beiden jeweils für kürzere Textpassagen keine Entsprechungen mehr bei Isidor finden lassen. Für zwei Passagen in der Musiktheorie konnte ich immerhin die Vorlage bei Guido von Arezzo aufspüren. Allerdings sind sie, da nur in einer $\mathrm{Hs}$. (V) vorhanden, wiederum interpolationsverdächtig (vgl. Summarium Heinrici, Band 1, S. XXV und XXVI). 
Ganz offensichtlich entwickelte der Summarium-Verfasser erst ab Buch III, d. h. mit der Beschreibung der Menschenwelt und ihrer Sachkultur - analog zu Isidors Büchern III-XX - seine eigene didaktische Intention der zweisprachigen Realienvermittlung. Sie könnte übrigens auf Grund eines speziellen Indizes auf einer Anregung durch Ekkehard IV von St. Gallen beruhen. Von diesem hatte der Summarium-Verfasser einen Übersetzungsversuch des bibelhebräischen und noch von Isidor als unübersetzbar erklärten „H os(i)anna“ wortwörtlich gegen Ende seines zweiten Buches übernommen und damit die Zweisprachigkeit seines Werkes eingeleitet ( $\mathrm{Hil}$ debr andt 1992).

Obwohl also die Isidor-Abhängigkeit durchaus gewollt und im Prosaprolog auch namentlich unterstrichen wird, hält sich der Summarium-Verfasser inhaltlich dennoch nicht an die Buchfolge bei Isidor, sondern gruppiert dessen Kapitel im Rahmen seines eigenen neuen inhaltlichen Konzeptes um und charakterisiert seine einzelnen Bücher durch entsprechende Ü berschriften:

Liber tertius: „De omni quod vi vit, sentit atque discernit.“

In zwanzig Kapiteln beschreibt er hier die belebte Welt mit Gott und Engeln als Überbau und sodann den Menschen in seinem sozialen Gefüge sowie seinen $\mathrm{K}$ örperteilen und fährt fort mit der Beschreibung der gesamten Tierwelt.

Liber quartus: „De his qu Ċvivunt per viriditatem.“

Hier wird die Pflanzenwelt beschrieben, beginnend mit dem Weinstock und endend mit dem Gemüse. Dass die Lebenskraft der Pflanzen auf ihrer viriditas ,Grünkraft' beruht, ist übrigens bei Hildegard von Bingen von zentraler Bedeutung und könnte wiederum deren Abhängigkeit vom Summarium bestätigen.

Liber quintus: „De omni quod movetur sed non vivificatur."

Diese Definition bezieht sich auf alles, was kosmisch und irdisch in Bewegung ist, ohne belebt zu sein. Auch die vom Menschen verursachte Bewegung wie das Reisen und der Ackerbau (cultura) gehört dazu.

Liber sextus: „De his qu Ċinveniuntur sub terra vel in terra.“

Hier finden die Steine und Metalle ihren Platz, auch hinsichtlich ihrer Nutzung durch den Menschen. 
Liber septimus: „De receptaculis hominum“ beschreibt die Städte, Bauwerke und W ohnungen bis hin zu den sieben W eltwundern.

Liber octavus: „In quo est de variis officiis hominum.“

Hier ist Raum zur Beschreibung von Völkerschaften und Berufsständen. Im Kapitel über die Völkerschaften bereitet die Nennung der Flavi ,valwun' im Verein mit den Ungarn, Bulgaren und Slawen für die Datierung des Summariums erhebliche Schwierigkeiten, denn diese Flavi (dt. Falben) müssen eindeutig mit dem turksprachigen Reitervolk der Komanen identifiziert werden, die 1070/71 Ungarn verwüsteten und erst durch dieses Ereignis ins Blickfeld des Summarium-Verfassers gelangen konnten. Die Entstehung des Summariums wäre dann frühestens um 1075 denkbar (W agner 1975). Diese Schlussfolgerung habe ich mir bisher nur zögerlich zu eigen gemacht, obwohl mir die damit verbundene Verlagerung von der Mitte ins ausgehende 11. J ahrhundert immer noch eine akzeptable Datierung ist.

Der Liber nonus "De vestimentis et alimentis et potibus et vasiis escariis“ beschreibt die Gegenstände des menschlichen Alltags und dabei vorrangig die Bekleidung und die Gefäße des täglichen Gebrauchs.

Im liber decimus „De bellis et de variis artificiis" schließlich kommt der W ortschatz der K riegsführung sowie aller sonstigen menschlichen Betätigungsfelder zur Geltung. Erst ganz am Ende wird Medizinisches behandelt, während dieses Thema bei Isidor bereits als erstes nach den septem artes stand.

Der letzte liber undecimus „D e interpretatione quorundam verborum secundum ordinem alphabeti" hat seine äußere Analogie zwar in dem auch bei Isidor mit semasiologischer Blickrichtung angelegten liber $X$, jedoch ist die alphabetische Wörtersammlung im Summarium eine gänzlich andere. Wahrscheinlich geht nur die von drei Handschriften überlieferte Kurzfassung noch auf den Summarium-Verfasser selbst zurück, drei Handschriften enthalten dagegen eine redaktionell stark erweiterte Langfassung, die dann auch in zehn Handschriften separat, $d$. h. ohne Anbindung an die zehn onomasiologischen Bücher des Summariums überliefert ist. Bemerkenswert ist dabei eine von den Buchstaben $\mathrm{A}$ bis $\mathrm{S}$ durchgehaltene Dreiteilung der Wörter als hebräisch, griechisch (beides in lateinischer Umschrift) und lateinisch, während in den Buchstaben T bis Z die Wörter der drei Sprachen untereinander vermischt sind. 
Ich möchte nun mein Augenmerk auf einige der vom Verfasser bewusst eingebrachten und über Isidor hinausgehenden aktualisierenden Zusätze richten, die sich sowohl in einigen eigen gestalteten Kapiteln als auch in den reichlichen Zusätzen zum Isidorstoff manifestieren:

Als echtes Kuriosum ist im Anschluss an die beiden Kapitel über die Tiere, die den Säugetieren entsprechen, ein als eigenes K apitel (III, 12) integriertes Poem anzusehen, betitelt „ut sine visu colorem vel sexum in agnis scias", ein offenbar dem Hirtenmilieu entstammendes Scherzgedicht, das für sich selbst sprechen mag:

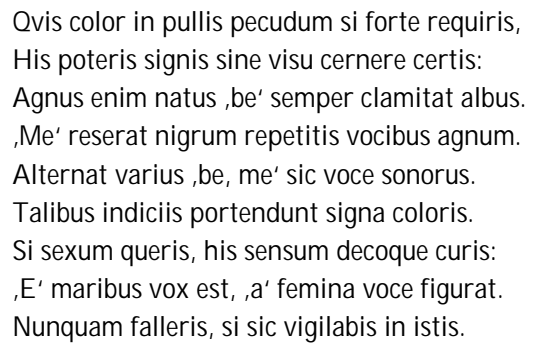

Sodann findet sich im Pflanzenbuch im Anschluss an zwei Bäumekapitel ein kurzes „De lignis“ (IV , 5), in dem eine Reihe von Baumnamen adjektivisch attribuiert werden als tennin holz, buochin holz, eichin holz, aber auch fremdländische Hölzer wie palmboumin, cederboumin, oleboumin holz. Diese Spezifizierungen reflektieren sicherlich ein sich differenzierendes Holzgewerbe.

Des weiteren erhalten auch die Baumfrüchte ein eigenes Kapitel (IV, 6), wobei auffällig ist, dass auch die Erdbeere darin vorkommt. allerdings werden die anderen B eerenfrüchte sonst überhaupt nicht erwähnt, so dass die Erdbeere nur hier unterzubringen war - immerhin aber doch in unmittelbarer Nachbarschaft mit der Maulbeere, mit der sie evtl. verglichen werden kann. Dass der Maulbeerbaum in anderen Quellen auch als Erdbeerbaum erscheint, sei hier nur am Rande erwähnt.

Am Ende des K apitels fügt der Verfasser dann noch eine differenzierte Beschreibung der Baumsäfte an, wobei neben pixpech, resina-harz auch gummi-kazzengolt vel fliet erscheint. Während letzteres schon ein althochdeutsch gut belegtes Wort für klebrig verdickten Baumsaft ist, war das hier verzeichnete Synonym kazzengolt ein schon von Rudolf $\mathrm{H}$ il debr and im DW B $(11,295)$ erkanntes mitteldeutsches Regionalwort des Summarium-Verfassers, das damit wesentlich früher belegt ist als die spätere, in der B ergmannssprache aufkommende Verwendung des Wortes in der Bedeutung ,gelber Glimmer' als Mineral. Ein an gleicher Stelle vorgenomme- 
ner Wortaustausch hat erst durch meine neueren Forschungen seine Aufklärung gefunden: Nur in der Darmstädter Handschrift des Summariums, deren Provenienz W. W egst ein (1985, S. 126ff.) im K loster Himmerod in der Eifel lokalisiert, wurde das W ort fliet durch flens ersetzt. Das wäre ein völlig obskures und deshalb bei Starck-W el I s (S. 164) auch mit einem $F$ ragezeichen versehenes Wort geblieben, wenn es nicht mehrfach als flins in gleicher Bedeutung bei Hildegard von Bingen belegt wäre. Damit ist das Wort neben seiner schon althochdeutschen Bedeutung "Feuerstein" (AhdWb. 3, 966f.) in der regional mitteldeutschen Eigenbedeutung ,Baumharz' bestätigt. Sie ist bisher lexikographisch nie erfasst, sondern nur indirekt im DWB 9, 1094 aus einem mitteldeutschen Vokabular (nach Dief enbach ) zitiert worden: gummi ,katzen clare flinsche'.

Äußerst rätselhaft bleiben schließlich in diesem Kapitel über Baumfrüchte die beiden letzten Einträge: ,elate dicuntur folia palmarum' und , agnosperma salbeiunbletir'. Beiden gemeinsam ist die Spezifizierung als folia bzw. -bletir. Aber während ein griechisches 미오 noch eindeutig in der Bedeutung ,Palmblätter' verifizierbar ist, ist bei agnosperma nur प0uा als griechisches Wort nachweisbar, das dann in lateinischer Umformung auch als agnus castus ein Synonym für vitex ,Abrahamsbaum' oder botanisch ungenau auch für salix ,Salweide' sein konnte; -sperma ,Samen' ist dagegen nur ein lateinisches Wort, für das es in unserem Zusammenhang nur eine mögliche Erklärung bei einem Zeitgenossen des SummariumVerfassers gibt: Constantinus Africanus schreibt: ,agnus castus calidus et siccus est in tertio gradu; sperma urit et desiccat" (MlatW b. 1, Spalte 396). Wieso der Summarium-V erfasser derartiges nun aber mit Salbeiblättern identifiziert, bleibt unerfindlich, und auch die Spekulation, dass er ahd. salbun- ,Salbei-' aus salhun- ,Salweiden-' verlesen haben könnte, erklärt noch nicht die Blätter statt der Samen.

Aus einem anderen Grund ist dieser singuläre und rätselhafte Summarium-Beleg aber noch besonders interessant: $\mathrm{Er}$ ist nämlich weitertradiert und wortwörtlich in den schwäbischen R edaktionszweig des "Vocabularius ex quo" (A 286.3) sowie in den "Vocabularius rerum" (D ief enbach $18^{\text {b) }}$ übernommen worden. Für die Rezeptionsgeschichte des Summariums ist das ein recht spektakulärer B efund!

Im Buch VII gibt es das eigenständige Kapitel III „De propriis nominibus civitatum", d. h. einen in seiner Gliederung und Herkunft sehr problemreichen Städtekatalog. W. WEGSTEINS Spurensuche (S. 40) führt hier auf die breit überlieferten und über J ahrhunderte ergänzten „Notitia Gallinarum" aus dem 5. J ahrhundert, in denen der Terminus civitas auf die Bedeutung „Bischofsstädte“ spezialisiert ist. Dazu passt im Summarium 
auch, dass als Vorlauf die fünf höherrangigen Patriarchate Konstantinopel, Alexandria, Antiochia, J erusalem und Aquileia benannt sind. Rom selbst aber bleibt als (vielleicht wegen der früh datierten Quelle noch nicht eindeutig fixierten) Spitze dieser Pyramide unerwähnt.

Das ist im Kapitel VIII, 2 „D e clericis et aliis sacris ordinibus“ dann allerdings anders, wo noch vor dem Patriarchen auch der bei Isidor wiederum fehlende papa-babest benannt wird, jedoch mit dem im ersten Teil merkwürdigen Interpretament „custos virginum vel apostolicus“ .

Während dieses benannte Kapitel dann im weiteren noch ganz nach Isidor gestaltet ist, wurde das folgende VIII, 3 „De variis officiis“ zweigeteilt, indem es zunächst nach Isidors Kapitel "De monachis“ den abbat, munich und einsidele benennt, aber dann die weiteren Bezeichnungen abbatissa, nunna sowie den loufari ,Wandermönch' und den klosinari zusätzlich einbringt. Ferner geht dem noch ein ganzer $\mathrm{K}$ atalog weltgeistlicher Ämter voraus: kanzilari, brievari, probest, dechan, kuster, mesinari, schuolmeister und canunich.

Am stärksten emanzipiert sich der Summarium-Verfasser gegenüber Isidor dann aber in der Umgestaltung und Erweiterung des von inm benannten Kapitels VIII, 8 „D e variis officiorum vocabulis vel operariis“. Dieses Kapitel war von Isidor mit der rein formalen Gliederung der Stichwörter nach dem Alphabet gestaltet worden, der Summarium-Verfasser hat seine ganz anderen Wörter dagegen mehr oder weniger konsequent nach Sachgruppen zu ordnen versucht. Eine von inm neu gebildete Sachgruppe stellte er dabei sogar an den Anfang des Kapitels: die Bediensteten einer mittelalterlichen Burg: truhtsazzo, scenko, kelnari, kamerari, bettekamerari, dresekamerari, phister, brotbecko, coch.

Neben den vielen anderen eingestreuten Zusätzen in diesem langen $K$ apitel, die hier im einzelnen nicht behandelt werden können, filtert der Verfasser einen Komplex sogar ganz heraus und gestaltet damit das zusätzliche Kapitel VIII, 9 „De notis vel vitiis in homine“ , d. h. die Benennungen der mit besonderen körperlichen Merkmalen und Gebrechen behafteten Menschen: scilihinter, nesilinter, lispenter, misel suhtiger, wazzersuhtiger, wobei sich an letzter Stelle makabrerweise auch das huorkint als ,filius scorti' befindet.

Ich schließe mit dem Hinweis auf eine Benennung, die in der heutzutage so ausufernden Sekundärliteratur zu Hildegard von Bingen, speziell jener, die für die Zielgruppe der auf Hildegard eingeschworenen Gesundköstler verfasst ist, immer wieder auftaucht, obwohl sie bei Hildegard nie eigens hervorgehoben und gebraucht wird. Der Summarium-V erfasser dagegen hat diese $\mathrm{W}$ endung nach der lateinischen $\mathrm{V}$ orgabe bei Isidor im Ka- 
pitel IX, 15 ,De mensis et escis': Dort steht neben den beiden lediglich auf das Backergebnis bezogenen Brot-Bezeichnungen irhabenbrot, derbibrot (vgl. H eyne, S. 268f.) als einziges ein auf eine spezielle Mehlsorte bezügliches Brot: ,siligineus panis dinkilinbrot'. Allerdings wird dieser wertvolle Frühbeleg von Dinkelbrot durch ein semasiologisches Problem belastet, denn nur der Summarium-V erfasser vertritt ganz singulär die semantische Äquivalenz von siligo und dinkil - auch in seinem Getreidekapitel (VI, 11), wo zudem noch die sonst allgemein auf Dinkel zu beziehende Gleichung spelta ,spelza' unmittelbar vorausgeht (vgl. H eyne, S. 271). Wenn siligo vom klassischen Latein her auch bei Isidor (XVII, 7) noch als besondere W eizenart, d. h. als ,genus tritici ... in pane species praecipua est' interpretiert wurde, so dürfte der Summarium-V erfasser von daher die Assoziation: feines B rotmehl = Dinkelmehl gehabt haben, ohne zu berücksichtigen, dass bei siligo zu seiner Zeit bereits der Bedeutungswandel zu ,Roggen" eingetreten war (D ief enbach $533^{\circ}$ ). Bei Hildegard gibt es dann in ihrer „Physica“ sowohl ein Kapitel siligo als auch ein Kapitel spelta. Für beide Wörter sind dann nur in der Florentiner Handschrift von späterer Hand auch die deutschen Äquivalente rogge und spelza vermerkt.

\section{Liter at ur und $\mathrm{N}$ achschlagewer ke}

AhdW b. 3 = Elisabeth K ar g-Gaster städt /Theodor Frings et al. (Hrsg., 1971-1985): Althochdeutsches Wörterbuch. Band 3. Berlin.

D ief enbach, Lorenz (1857): Glossarium latino-germanicum mediae et infimae aetatis. Frankfurt a. M. (Neudruck Darmstadt 1968).

DWB 9 und $11=\mathrm{J}$ acob und Wilhelm Grimm et al. (Hrsg.): Deutsches Wörterbuch. Band 9 (1935) (alte Zählung IV , I, 6); Band 11 (1873) (alte Zählung V). Leipzig (Neudruck mit neuer Zählung München 1984).

DWF 9.2 = J ürgen Erich Sch midt// oachim H er r gen (Hrsg., 2002): Deutsche W ortforschung in europäischen Bezügen. Band 9.2: Reiner Hildebrandt: Gesammelte Beiträge zur Germanistik II. Gießen.

H eyne, Moriz (1901): Deutsche Hausaltertümer. Bd. 2: „Nahrung“. Leipzig.

H il debr andt, Reiner (1986): Rezension zu Werner Wegstein (1985). In: ZfdA 115, S. 120129 (Nachdruck in DWF 9.2, S. 15-24).

H il debr andt, Reiner (1992): U uillechomô! Ekkehards IV. beliebte Grußformel. In: Harald Burger et al. (Hrsg., 1992): Verborum amor. Studien zur Geschichte und Kunst der deutschen Sprache (Festschrift Sonderegger). Berlin, S. 238-248 (Nachdruck in DWF 9.2, S. 81-91).

H il debr andt, Reiner (1995): Summarium Heinrici. In: Burghart W achinger et al. (Hrsg., 1995): Die deutsche Literatur des Mittelalters. Verfasserlexikon. Band 9. Berlin, Sp. 510-519. 
H il debr andt, Reiner (1996a): Summarium Heinrici: Das Lehrbuch der Hildegard von Bingen. In: Ernst Bremer, Reiner Hildebrandt (Hrsg., 1996): Stand und Aufgaben der deutschen Dialektlexikographie II. Berlin, S. 89-110 (Nachdruck in DWF 9.2, S. 103125).

H il debr andt, Reiner (1996b): Zum Wortschatz der Hildegard von Bingen (Essigkalb, Denemarcha, ziergerne). In: Studier i talesprogsvariation og sprogkontakt (Festskrift Inger E j skj ær ). Kopenhagen, S. 124-134 (Nachdruck in DWF 9.2, S. 125-136).

Isidor = Lindsay, W. M. (Hrsg., 1911): Isidori Hispalensis E piscopi Etymologiarum sive Originum libri XX, Tomus I HI. Oxford (Nachdruck Oxford 1957, 1962).

MlatWb. 1 = Bayerische Akademie der Wissenschaften (Hrsg., 1967): Mittellateinisches Wörterbuch. Band 1. München.

Reynol ds, L. D. (1965): The Medieval tradition of Seneca's Letters. Oxford.

St ar ck-W el I s = St arck, Taylor/W el I s, J ohn C. (Hrsg.) (1990): Althochdeutsches Glossenwörterbuch. Heidelberg.

Summarium Heinrici = Reiner H il debr andt (Hrsg.) (1974/1982/1995): Summarium Heinrici. Textkritische Ausgabe. Band 1 und 2, Register der deutschen Glossen. Band 3. Berlin.

Vocubularius ex quo = K laus Gr ubmül I er /Bernhard Schnel I et al. (Hrsg.) (1988/89): Vocabularius ex quo. Ü berlieferungsgeschichtliche Ausgabe. Band 1-5. Tübingen.

W agner , Norbert (1975): Zur Datierung des Summarium Heinrici. In: ZfdA. 104, S. 118- 126.

W egstein, Werner (1985): Studien zum Summarium Heinrici. Die Darmstädter Hs. 6. Tübingen. 